\title{
Designing A Social VR Clinic for Medical Consultations
}

$\begin{array}{ll}\text { Jie Li } & \text { Huib de Ridder } \\ \text { CWI } & \begin{array}{l}\text { TU Delft } \\ \text { Science park 123 }\end{array} \\ \begin{array}{l}\text { Landbergstraat 15 } \\ \text { j.li@cwi.nl }\end{array} & \text { 2628 CE Delft, NL } \\ & \text { H.deRidder@tudelft.nl } \\ & \\ & \\ \text { Guo Chen } & \text { Pablo Cesar } \\ \text { CWI } & \text { CWI \& TU Delft } \\ \text { Science park 123 } & \text { Science park 123 } \\ \text { 1098XG Amsterdam, NL } & \text { 1098XG Amsterdam, NL } \\ \text { g.chen1995123@gmail.com } & \text { P.S.Cesar@cwi.nl }\end{array}$

Permission to make digital or hard copies of part or all of this work for personal or classroom use is granted without fee provided that copies are not made or distributed for profit or commercial advantage and that copies bear this notice and the full citation on the first page. Copyrights for third-party components of this work must be honored. For all other uses, contact the owner/author(s).

Copyright held by the owner/author(s).

CHI'20 Extended Abstracts, April 25-30, 2020, Honolulu, HI, USA ACM 978-1-4503-6819-3/20/04.

https://doi.org/10.1145/3334480.3382836

\begin{abstract}
Social Virtual Reality (VR) invites multiple users to "interact" in a shared immersive environment, which creates new opportunities for remote communication, and can potentially be a new tool for remote medical consultations. Using knee osteoarthritis consultation as a use case, this paper presents a social VR clinic that allows patients to consult a nurse represented as a virtual avatar with head, upper body and hands visible. We started with an ethnographic study at a hospital with three medical professionals and observed three patient consultation sessions to map the patient treatment journey (PTJ) and distill design requirements for social VR consultation. Based on the results of the study, we designed and implemented a social VR clinic to meet the identified requirements. Our work expands on the potential of social VR to help reshape patient treatment by reducing the workload of medical staff and the travel time of patients. In the future, we plan to conduct user studies to compare face-to-face (F2F) with social VR consultations.
\end{abstract}

\section{Author Keywords}

Virtual reality; social VR; VR consultation; telehealth; user studies

\section{CCS Concepts}

•Human-centered computing $\rightarrow$ User studies; Virtual reality; 


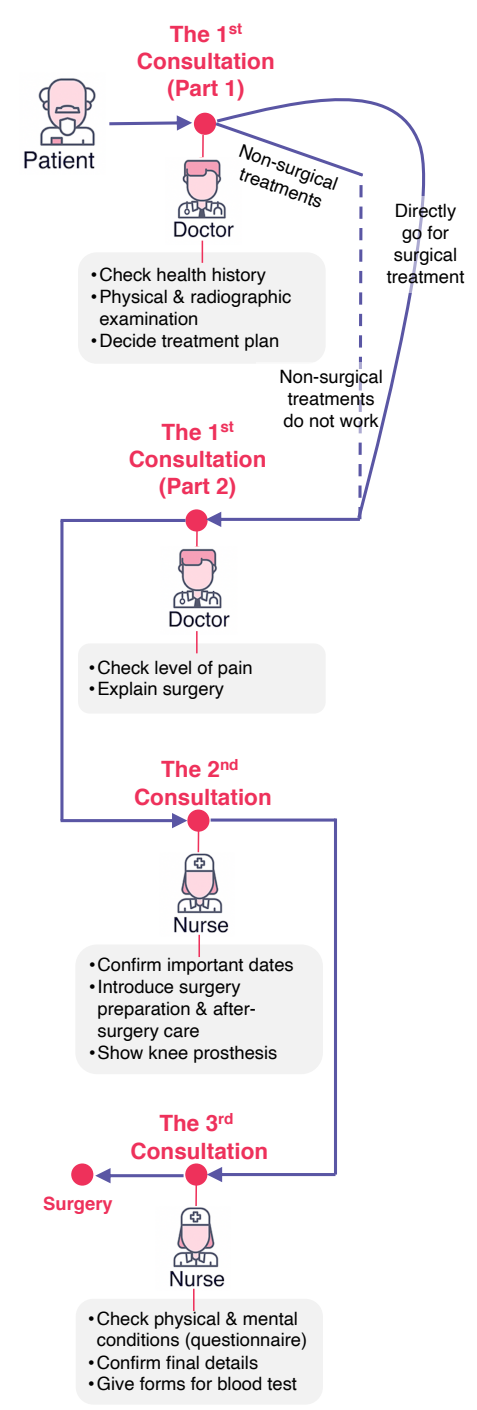

Figure 1: The patient treatment journey for knee osteoarthritis.

\section{Introduction}

Virtual Reality (VR) in healthcare has long been envisioned as a promising technology that resembles face-to-face (F2F) communication between patients and medical professionals $[11,15,23]$. With social VR technologies, multiple users can "meet" in a shared, immersive virtual environment and interact with the virtual representations of each other [12]. So far, many VR healthcare applications have been developed for medical training [28], psychological consultation [29] and remote (psycho)therapy [6]. A national survey (2006-2017) in US found that the time people spent traveling to healthcare services was the longest compared to other professional services (e.g., legal/governmental services). The time spent traveling and waiting for healthcare was over $50 \%$ of the time actually spent receiving care [26]. Beside the time cost, traveling is painful for the patients who have disabilities or suffer from chronic disease.

Knee osteoarthritis is a condition in which the natural cushioning (i.e., cartilage) between joints wears away and patients suffer from the pain caused by the bones rub against one another [9]. We chose knee osteoarthritis treatments as a use case, aiming at reducing the traveling time of patients and the workload of medical staff. This paper has two contributions: (1) gather requirements for social VR remote consultation; (2) Design and implement a social VR clinic to support remote consultation based on the requirements. We conducted an ethnographic study with medical professionals and patients in a hospital. The results illustrated a detailed patient treatment journey, and showed the heavy workload of medical professionals and their communication problems with patients. We defined four main requirements according to the study results. The implemented social VR clinic addressed the requirements by simulating the real hospital environment, offering a walk-in virtual surgery room and realistic 3D anatomy models to support the re- mote consultation. Our work demonstrates the potential of social VR as a new remote communication tool.

\section{Related Work}

To properly manage health, it is essential that patients know about their health. However, communication between medical staff and patients is often ineffectual, thereby limiting the patients' knowledge of their health and treatment options [34]. Ventres et al. [36] introduced "shared presence", describing "doctors and patients entering into a deep sense of trust and respect" that facilitates the treatment, and supports effective consultations. Aelbrecht et al. [2] pointed out that patients' needs may differ: some emphasised the emotional support, while others found problem-focused discussions important. Riedl and Schüßler [27] stressed that participatory decision making led to improved doctor-patient relationship and interpersonal exchange [3]. Kelly et al. [17] found that a warm and empathic treatment atmosphere is important. These factors need to be well considered when developing technologies to support medical consultations.

Remote medical consultation is cost-effective, providing similar quality as F2F consultation, and erases geographic distance [8]. VR technologies are considered as an extension to existing communication tools (e.g., video conferencing), and are explored as new applications for healthcare, including disseminating health information, providing remote (psycho) therapies [1], and training medical professionals [22]. Medical consultations in VR are distinguished from video consultations by their capacity to portray 3D spatial information [35], to exploit users' natural behaviors, and to immerse users in the virtual world. VR can use avatars to offer appearance, gestures, directional voice, and ability to interact with the environment and virtual artifacts [13]. Walia et al. [37] see VR as a supplemental solution to the nursing shortage and to assist patients with disabilities. 


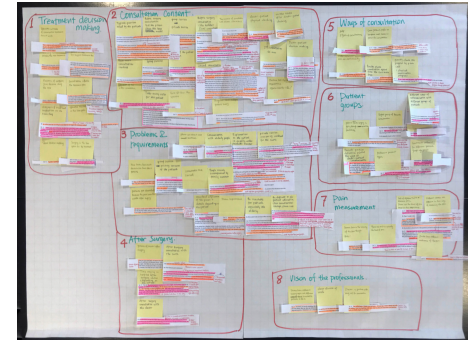

Figure 2: The categorization of the open coded transcripts

\section{Methods: An Ethnographic Study}

We selected knee osteoarthritis treatment as a use case to design the social VR clinic. To get an overview of the treatment process and the content communicated, an ethnographic study [4, 38] was conducted in the Reinier de Graaf hospital located in Delft, The Netherlands. The study has two parts: (1) one-to-one semi-structured interviews [20] with three medical professionals, and (2) observational studies at three nurse-patient consultations [16, 5].

\section{Medical Professionals}

Three medical professionals participated in the semi-structured interviews, including two surgeons (the doctors) and one consultant (the nurse). Both doctors have over ten years of experience in the treatment of knee osteoarthritis. They perform surgery, discuss with patients about treatment plans, and examine their recovery progress. The nurse works closely with the two doctors, and prepares patients for the surgery. The nurse that participated in the observational studies is the same person as in the interviews.

\section{Procedure}

The interviews were conducted at the office of medical professionals. Each interview lasted one hour with prepared in terview questions illustrated in a booklet. The professionals were asked to explain their answers while filling in the booklet. In this way, we expected to guide them to tell concrete stories rather than provide abstract answers to the questions, helping obtain latent information behind the conversations [30]. Besides the interviews, observational studies were conducted by two researchers during three patient-

nurse consultations. The interviews and observations were both audio recorded and later on transcribed.

\section{Data Collection and Analysis}

Three types of data were collected in the studies: (1) transcribed audio recordings; (2) the filled booklets by the med- ical professionals and the notes taken during the observations; and (3) the printed materials from the hospital (e.g., a patient manual about the surgery.)

The transcripts and notes were independently coded by two researchers, following an inductive open coding approach [32]. The overlapping codes from the two researchers were kept for later categorization. Keeping or discarding the nonoverlapping codes were decided by the two researchers. Afterwards, the selected codes were sorted into two main categories: (1) patient treatment journey, and (2) problems and opportunities for medical consultation.

\section{Results}

This section presents the results of the ethnographic study. The three medical professionals are labelled as M1-M3.

\section{Patient Treatment Journey}

For patients who need knee replacement surgery, there are three consultations (Figure 1). All patients start with the first consultation with the doctor, for examination and making decisions about the treatment. When the patient needs to have the surgery, a second and third consultations will be scheduled with the nurse.

The first consultation. The doctor meets the patient in person to do the medical examinations in this consultation. M2 described the main tasks involved, "I do three things: asking the history, doing a physical examination and having the patient examined by the $X$-ray." The doctor explains the procedure and risks of the surgery, and shares the decision about the treatment with the patient. "I tell them [the patients] about the process, complications and risks of the surgery. We made decisions together (M1)."

The second consultation. Most patients start the treatment with non-surgical treatments (e.g., medications and injec- 
tions). As mentioned by M2, "Here in the hospital, we only operate $15-20 \%$ of the patients. For the other $80-85 \%$, we treat them through injections, physiotherapy and medications. "When the patient needs surgery, then we schedule the second consultation with the nurse 6-7 weeks in advance. The second consultation is a 20 -minute $Q \& A$ session with the nurse, where the preparation of the surgery is explained in details. Patients are encouraged to ask questions during this consultation "I show them [the patients] the knee prosthesis model, and talk about the surgery. I always make sure the patients feel the weight of the prosthesis. If they do not have many questions, I will point out a few important things for them to remember (M3)." The second consultation involves a lot of conversations and physical interactions.

The third consultation. The third consultation takes about 45 minutes, happening a few days before the surgery, to confirm the the surgery details, and to ask the patient to fill in a comprehensive questionnaire about their physical and mental conditions. As M3 told us, "We need to make sure when the patient comes to the surgery, everything is recorded. The surgeon and the ward are prepared. We ask everything in the questionnaire: their physical, mental conditions, whether they have people to take care of them, their home environment..." The third consultation does not involve much verbal or physical interaction.

\section{Problems \& Opportunities for Medical Consultation}

Communication difficulties. The three medical professionals pointed out that communicating with the elderly is difficult. "I think the biggest problem we are facing now is the communication with the elderly. Most of the patients are above 80 and some of them have dementia (M3)." The medical professionals also have limited time to repeat the explanations, but they try to explain things in an under- standable manner, avoiding using medical jargons. M3 told us, "We tell them about the surgery in an easy way. If we need to use medical terms, we explain them well to make sure they understand them. We show them the prosthesis and tell them how the surgeon is going to operate on their knee."

Heavy workload. The medical professionals complained about their heavy workload. Due to the privacy protection, the consultation is a one-to-one session with every patient. M3 told us, "It takes a lot of time, and is boring for me to repeat the same story six times a day. We had the consultation in groups before, but we found patients reluctant to talk about their problems or ask questions in front of others. So, we changed it to private sessions." Another aspect of the workload is that the medical professionals must be

"approachable by the patients all the time (M3)".

Social VR: new opportunity. The medical professionals occasionally use video conferencing to answer minor questions from patients, but they found it difficult to explain and visualize things there. "We tried to do it remotely, but it turned out to be unpractical, because we had difficulties to show visuals and documents (M3)." The medical professionals all agreed that social VR can provide distinguished advantages compared to video consultations, such as displaying 3D animated information, and allowing users to use gestures, hear directional voice, and interact with the virtual objects. "I can see this [social VR clinic] is plausible in many ways, like visualizing the medical knowledge and having people meet in the same [virtual] space (M2)." The medical professionals also see social VR as a supplemental solution to the nursing shortage and to assist patients with disabilities, as M3 said, "If we can record the explanations and visualizations in social VR, and let the VR nurse to repeat the it. This can save me and the patient a lot of time." 

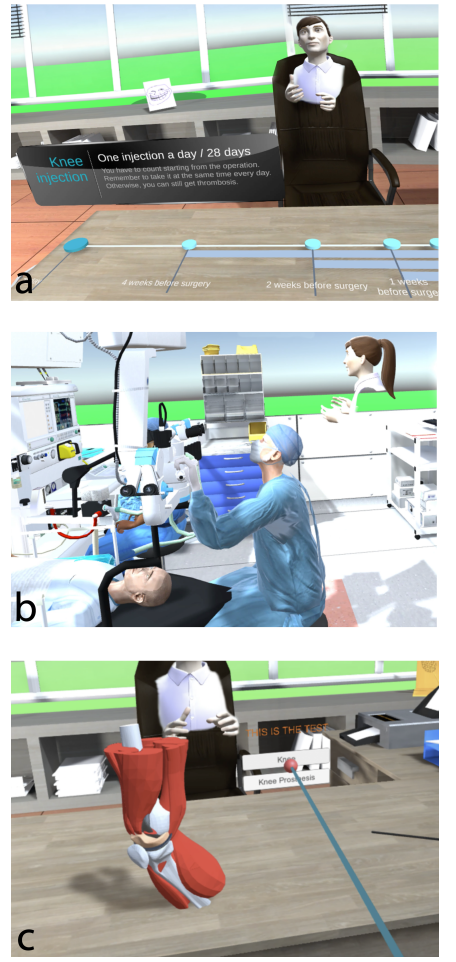

Figure 3: The social VR clinic: (a) visualized surgery preparation timeline; (b) 3D "walk-in" surgery room; (c) 3D interactive knee anatomy and prosthesis models
Design \& Implementation of a Social VR Clinic We decided to focus on the second consultation of the PTJ to design the social VR clinic. This consultation has three main activities (Figure 4): (1) explain how patients should prepare for the surgery and stress the important information (e.g., dates, medications); (2) show a video about the surgery room; (3) explain the surgery process using the knee prosthesis and ask patients to feel its weight.

\section{Design Requirements}

Based on the ethnographic study, and the related work, we defined the requirements for a social VR clinic (RQMT 1RQMT 4):

RQMT 1: Replicate the three main activities of a F2F medical consultation;

RQMT 2: Enable verbal and non-verbal (e.g., spoken, visual, gestural) communications;

RQMT 3: Provide visualizations and animations of the medical knowledge to assist the communication;

RQMT 4: Enable record and replay of the consultation to reduce the repetition and workload.

\section{Implementation: Addressing the Requirements}

A combination of spoken and visual information is easier for patients to remember than only verbally explained information $[18,33]$. Therefore, the designed social VR clinic maximizes information visualizations (RQMT 3). The social VR clinic (1) visualizes the preparation timeline and explains the medical jargon; (2) allows the patient to "walk into" a 3D virtual surgery room to "meet" the medical staff, and (3) enables the patient to interact with an animated 3D knee anatomy model and a knee prosthesis to see what the differences are before and after the surgery. The three activities (Figure 3 ) are well replicated in social VR clinic (RQMT 1). The nurse is represented by an avatar, which captures the real-time head, hands, mouth and body move-

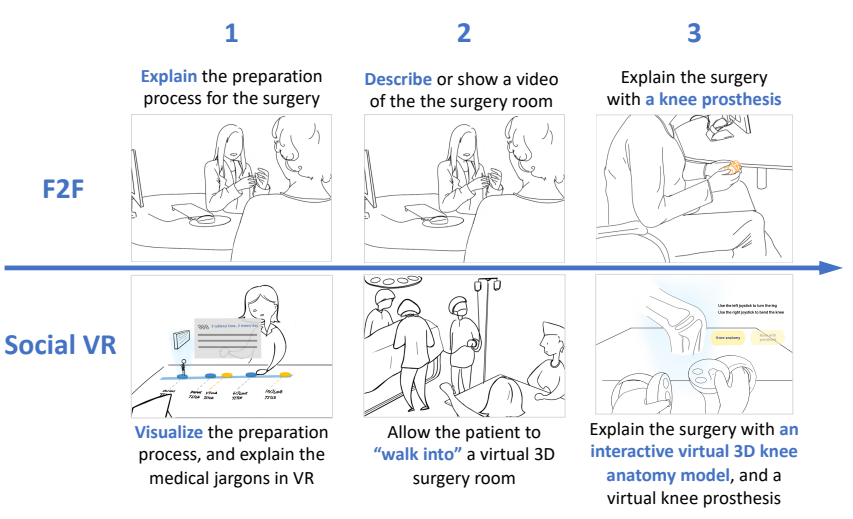

Figure 4: Comparing the three main activities of the second consultation in F2F and social VR conditions

ments (RQMT 2). The recorded social VR consultation can be replayed and shared to the patient (RQMT 4).

The prototype is implemented in Unity ${ }^{1}$. Oculus Integration for Unity is also applied ${ }^{2}$, which has pre-built functions, such as interfaces for controlling VR cameras. The knee and the prosthesis model implementations were adapted based on professionally 3D scanned medical models from Thingiverse ${ }^{3}$. We added the material layer and motion to the models in Unity and incorporated them into the prototype. The surgery room is based on an Asset from the Unity Store ${ }^{4}$, including a set of realistic medical devices, furniture objects and animations.

\footnotetext{
${ }^{1} \mathrm{https}: / /$ unity.com, retrieved on Aug. 26, 2019

${ }^{2}$ https://developer.oculus.com/downloads/package/unity-integration/, retrieved on Aug. 26, 2019

${ }^{3} \mathrm{https}: / / w w w . t h i n g i v e r s e . c o m / t h i n g: 340254$, retrieved on Aug. 26, 2019

${ }^{4}$ https://assetstore.unity.com/packages/3d/props/interior/operating room-18295, retrieved on Aug. 26, 2019
} 


\section{Discussion \\ Limitations \& Benefits of Social VR}

The implemented social VR clinic was based on the $2^{\text {nd }}$ consultation, since it involves the most verbal communication, and does not require medical examination (e.g., draw blood). The current social VR clinic is only for patients who have been previously examined by medical professionals in F2F settings. Some researchers expressed their concerns that remote consultations may be clinically risky, and bring significant technical, logistical and regulatory challenges (e.g., [10]). Therefore, we do not recommend using social VR as the primary means of contact between medical staff and patients, but as an extension and augmentation for remote communication. As an augmentation, social VR provides many benefits. First, it immerses the users in the same virtual world, providing realistic and co-presence experience [19]. Second, it uses virtual representations to offer embodiment experiences to users, and abilities to interact with the virtual environment and artifacts [13]. Third, it enables the sense of social connectedness [19] and allows people to see and feel from other person's perspective [31].

\section{Virtual Representations \& Privacy}

We implemented a simple non-realistic avatar to represent the users, with only head, upper body and hands visible. This avatar can assist the spoken and gestural communication, but may fail to emotionally engage the users. The medical professionals raised their concerns on the type of virtual representations used in social VR: To use the realistic ones to help them better diagnose or to use the nonrealistic ones to protect the privacy of patients? Some research proposed real-time photo-realistic human representations in VR (e.g., [25]), or worked on HMD removal (e.g., [39]), trying to make the user face visible to enhance the presence and immersion. However, the trade-off between the realism of the user representations and the privacy protection should be considered in future work.

\section{Multisensory Experiences in VR}

Multisensory experiences can enhance users' presence and immersion, and enable them to have better task performances in a virtual environment [14, 24]. In our study, the medical professionals stressed the necessity of multisensory feedback in VR. For instance, to help patients build a proper expectation of the surgery, they must feel the weight of the prosthesis that is going to be implanted in their knees. However, the weight simulation is not implemented in our prototype. Many studies have investigated the haptics and weight simulations in VR (e.g., [7, 21]). In future work, we are interested in exploring haptic experiences in VR, especially investigating how to accurately simulate the weight of a virtual object.

\section{Conclusion}

In this early work, we designed and implemented a social VR clinic for patients to remotely consult medical professionals. The goal is to enable patients to travel fewer times but still receive good quality consultations. The implemented social VR clinic simulates a consultation office, a surgery room, and $3 \mathrm{D}$ anatomy models. Our work expands on the potential of social VR to help reshape remote medical consultations. In the future, we plan to conduct user studies, to compare social VR consultation with the F2F one. We will also continuously explore use cases for socia VR (e.g., engaging patients with dementia), improve the user representations and investigate haptic experiences, such as feeling or perceiving the weight of virtual objects.

\section{Acknowledgement}

This work is funded by the European Commission H2020 program, under the grant agreement 762111, VRTogether, 
http://vrtogether.eu/

\section{REFERENCES}

[1] Michelle Aebersold and Dana Tschannen. 2012. Using virtual simulations in second life for teaching and learning in nursing education. Engaging the Avatar: New Frontiers in Immersive Education (2012), 311.

[2] Karolien Aelbrecht, Michela Rimondini, Jozien Bensing, Francesca Moretti, Sara Willems, Mariangela Mazzi, lan Fletcher, and Myriam Deveugele. 2015. Quality of doctor-patient communication through the eyes of the patient: variation according to the patient's educational level. Advances in Health Sciences Education 20, 4 (2015), 873-884.

[3] Jeffrey A Alexander, Larry R Hearld, Jessica N Mittler, and Jillian Harvey. 2012. Patient-physician role relationships and patient activation among individuals with chronic illness. Health services research 47, 3pt1 (2012), 1201-1223.

[4] Michael Angrosino. 2007. Doing ethnographic and observational research. Sage.

[5] K Louise Barriball and Alison While. 1994. Collecting data using a semi-structured interview: a discussion paper. Journal of Advanced Nursing-Institutional Subscription 19, 2 (1994), 328-335.

[6] Penny E Bee, Peter Bower, Karina Lovell, Simon Gilbody, David Richards, Linda Gask, and Pamela Roach. 2008. Psychotherapy mediated by remote communication technologies: a meta-analytic review. BMC psychiatry 8, 1 (2008), 60.

[7] Inrak Choi, Heather Culbertson, Mark R Miller, Alex Olwal, and Sean Follmer. 2017. Grabity: A wearable haptic interface for simulating weight and grasping in virtual reality. In Proceedings of the 30th Annual ACM
Symposium on User Interface Software and Technology. ACM, 119-130.

[8] Alessandro Di Cerbo, Julio Cesar Morales-Medina, Beniamino Palmieri, and Tommaso lannitti. 2015. Narrative review of telemedicine consultation in medical practice. Patient preference and adherence 9 (2015), 65.

[9] David T Felson, Allan Naimark, Jennifer Anderson, Lewis Kazis, William Castelli, and Robert F Meenan. 1987. The prevalence of knee osteoarthritis in the elderly. The Framingham Osteoarthritis Study. Arthritis \& Rheumatism: Official Journal of the American College of Rheumatology 30, 8 (1987), 914-918.

[10] Trisha Greenhalgh, Sara Shaw, Joseph Wherton, Shanti Vijayaraghavan, Joanne Morris, Satya Bhattacharya, Philippa Hanson, Desirée

Campbell-Richards, Seendy Ramoutar, Anna Collard, and others. 2018. Real-world implementation of video outpatient consultations at macro, meso, and micro levels: mixed-method study. Journal of medical Internet research 20, 4 (2018), e150.

[11] Walter Greenleaf. 2016. How VR technology will transform healthcare. In ACM SIGGRAPH 2016 VR Village. ACM, 5.

[12] Paul Heidicker, Eike Langbehn, and Frank Steinicke. 2017. Influence of avatar appearance on presence in social VR. In 2017 IEEE Symposium on 3D User Interfaces (3DUI). IEEE, 233-234.

[13] Trevor Jamieson, Ross Wallace, Katie Armstrong, Payal Agarwal, Bailey Griffin, Ivy Wong, and S Bahtia. 2015. Virtual care: a framework for a patient-centric system. Toronto: Women's College Hospital Institute for Health Systems Solutions and Virtual Care (2015). 
[14] Sarah Jones and Steve Dawkins. 2018. The sensorama revisited: evaluating the application of multi-sensory input on the sense of presence in 360-degree immersive film in virtual reality. In Augmented reality and virtual reality. Springer, 183-197.

[15] K-F Kaltenborn and O Rienhoff. 1993. Virtual reality in medicine. Methods of information in medicine 32, 05 (1993), 407-417.

[16] Barbara B Kawulich. 2005. Participant Observation as a Data Collection Method. (2005).

[17] John M Kelley, Anthony J Lembo, J Stuart Ablon, Joel J Villanueva, Lisa A Conboy, Ray Levy, Carl D Marci, Catherine Kerr, Irving Kirsch, Eric E Jacobson, and others. 2009. Patient and practitioner influences on the placebo effect in irritable bowel syndrome. Psychosomatic medicine 71, 7 (2009), 789.

[18] Roy PC Kessels. 2003. Patients' memory for medical information. Journal of the Royal Society of Medicine 96, 5 (2003), 219-222.

[19] Jie Li, Yiping Kong, Thomas Röggla, Francesca De Simone, Swamy Ananthanarayan, Huib de Ridder, Abdallah El Ali, and Pablo Cesar. 2019. Measuring and understanding photo sharing experiences in social Virtual Reality. In Proceedings of the $2019 \mathrm{CHI}$ Conference on Human Factors in Computing Systems. ACM, 667.

[20] Robyn Longhurst. 2003. Semi-structured interviews and focus groups. Key methods in geography 3 (2003), 143-156.

[21] Pedro Lopes, Sijing You, Lung-Pan Cheng, Sebastian Marwecki, and Patrick Baudisch. 2017. Providing haptics to walls \& heavy objects in virtual reality by means of electrical muscle stimulation. In Proceedings of the $2017 \mathrm{CHI}$ Conference on Human Factors in Computing Systems. ACM, 1471-1482.

[22] Elhassan Makled, Amal Yassien, Passant Elagroudy, Mohamed Magdy, Slim Abdennadher, and Nabila Hamdi. 2019. PathoGenius VR: VR medical training. In Proceedings of the 8th ACM International Symposium on Pervasive Displays. ACM, 31.

[23] Daniel G McDonald and Michael A Shapiro. 1992. I'm Not a Real Doctor, but I Play One in Virtual Reality: Implications of Virtual Reality for Judgments about Reality. Journal Of Communication 42, 4 (1992).

[24] Marianna Obrist, Elia Gatti, Emanuela Maggioni, Chi Thanh Vi, and Carlos Velasco. 2017. Multisensory experiences in HCI. IEEE MultiMedia 24, 2 (2017), 9-13.

[25] Sergio Orts-Escolano, Christoph Rhemann, Sean Fanello, Wayne Chang, Adarsh Kowdle, Yury Degtyarev, David Kim, Philip L Davidson, Sameh Khamis, Mingsong Dou, and others. 2016. Holoportation: Virtual 3d teleportation in real-time. In Proceedings of the 29th Annual Symposium on User Interface Software and Technology. ACM, 741-754.

[26] Corwin N. Rhyan. 2019. Travel and Wait Times Are Longest for Health Care Services. (2019). https://altarum.org/travel-and-wait

[27] David Riedl and Gerhard Schüßler. 2017. The influence of doctor-patient communication on health outcomes: a systematic review. Zeitschrift für Psychosomatische Medizin und Psychotherapie 63, 2 (2017), 131-150. 
[28] Giuseppe Riva. 2014. Medical clinical uses of virtual worlds. In The Oxford handbook of virtuality. Oxford University Press, New York, 649-665.

[29] Giuseppe Riva, Antonios Dakanalis, and Fabrizia Mantovani. 2015. Leveraging psychology of virtual body for health and wellness. The Handbook of the Psychology of Communication Technology. Chichester UK: John Wiley \& Sons, Ltd (2015), 528-47.

[30] Liz Sanders. 2012. Convivial Toolbox: Generative Research for the Front End of Design pdf by. (2012).

[31] Donghee Shin. 2018. Empathy and embodied experience in virtual environment: To what extent can virtual reality stimulate empathy and embodied experience? Computers in Human Behavior 78 (2018), 64-73.

[32] David R Thomas. 2006. A general inductive approach for analyzing qualitative evaluation data. American journal of evaluation 27, 2 (2006), 237-246.

[33] Ann M Thomson, Susan J Cunningham, and Nigel $P$ Hunt. 2001. A comparison of information retention at an initial orthodontic consultation. The European Journal of Orthodontics 23, 2 (2001), 169-178.

[34] Anh N Tran, Paul Haidet, Richard L Street Jr, Kimberly J O'Malley, Frank Martin, and Carol M Ashton. 2004. Empowering communication: a community-based intervention for patients. Patient education and counseling 52, 1 (2004), 113-121.

[35] Jose E Venson, Jean Berni, Carlos S Maia, A Marques da Silva, Marcos d'Ornelas, and Anderson Maciel. 2016. Medical imaging VR: can immersive 3D aid in diagnosis?. In Proceedings of the 22nd ACM Conference on Virtual Reality Software and Technology. ACM, 349-350.

[36] William B Ventres and Richard M Frankel. 2015. Shared presence in physician-patient communication: A graphic representation. Families, Systems, \& Health 33, 3 (2015), 270.

[37] Nitin Walia, Fatemeh Mariam Zahedi, and Hemant Jain. 2017. Potential of Virtual Worlds for Nursing Care: Lessons and Outcomes. OJIN: the Online Journal of Issues in Nursing 23, 1 (2017).

[38] Harry F Wolcott. 1999. Ethnography: A way of seeing. Rowman Altamira.

[39] Yajie Zhao, Qingguo Xu, Weikai Chen, Chao Du, Jun Xing, Xinyu Huang, and Ruigang Yang. 2019.

Mask-off: Synthesizing face images in the presence of head-mounted displays. In 2019 IEEE Conference on Virtual Reality and 3D User Interfaces (VR). IEEE, 267-276. 